\title{
Interaction of Magnetic Islands with Turbulent Electron Temperature Fluctuations in DIII-D and in GENE Non-Linear Gyrokinetic Simulations
}

\author{
L. Bardóczi, ${ }^{1,2, a)}$ C. Sung, ${ }^{2,3}$ A. Bañón Navarro, ${ }^{4}$ T. L. Rhodes, ${ }^{2}$ T. A. Carter, ${ }^{2}$ and F. Jenko ${ }^{4,5}$ \\ 1) General Atomics, P. O. Box 85608, San Diego, California 92186-5608, USA \\ ${ }^{2)}$ University of California, Los Angeles, California 90095, USA \\ ${ }^{3)}$ Lam Research Corporation, Fremont, California 94538, USA \\ 4) Max Planck Institute for Plasma Physics, Garching 85748, Germany \\ ${ }^{5)}$ University of Texas at Austin, Austin, Texas 78712, USA
}

(Dated: 25 November 2019)

We present localized measurements of interactions between Neoclassical Tearing Modes (NTMs) and turbulent electron temperature fluctuations $\left(\widetilde{\mathrm{T}}_{\mathrm{e}}\right)$ in the expected $k_{\theta} \rho_{s}=0-0.5$ wave-number range of the Ion Temperature Gradient (ITG) instability measured via Correlation Electron Cyclotron Emission $\left(k_{\theta}\right.$ and $\rho_{s}$ are the poloidal wave-number and ion-sound Larmor radius, respectively). Comparison to GENE gyrokinetic simulations, shows qualitative agreement with $\widetilde{\mathrm{T}}_{\mathrm{e}}$ being reduced (increased) inside (outside) of the islands due to modified local gradients, and $\widetilde{T}_{e}$ being higher (lower) in the region outside the island where lower (higher) flow shear is expected due to radially asymmetric island shape. These results are consistent with previously reported modifications of long and intermediate wavelength turbulent density fluctuations and cross-field electron thermal diffusivity reduction at the O-point of magnetic islands. Interestingly, a $30 \%$ increase of $\widetilde{T}_{e}$ is detected at the NTM rational surface when the island width is a few times $\rho_{s}$, which is replicated by GENE through zonal flow damping due to the destroyed magnetic field topology of the NTM rational surface.

\section{INTRODUCTION}

Neoclassical Tearing Modes (NTM) are large-scale resistive magnetohydrodynamic (MHD) instabilities that form magnetic islands on low order rational flux surfaces of tokamak plasmas. $m / n=2 / 1$ NTMs are important as they can severely degrade the plasma confinement and can lead to plasma termination ${ }^{1}$ ( $m$ and $n$ are the poloidal and toroidal mode numbers, respectively). The effect of magnetic islands on small-scale turbulence and the impact of turbulence on NTM stability have been an active field of theoretical research ${ }^{2-19}$. Recently, a number of experiments studied the effect of stationary magnetic islands on low- and intermediate-k turbulent density fluctuations ( $\widetilde{\mathrm{n}}$ ) with Far Infrared Scattering (FIR), Beam Emission Spectroscopy (BES), Doppler Backscattering (DBS) and Interferometry ${ }^{20-24}$. Measurements of $\widetilde{\mathrm{n}}$ via BES, DBS and FIR across $m / n=2 / 1$ NTM magnetic islands were reported recently in DIII-D ${ }^{25-28}$. These experiments reported reduced $\widetilde{n}$ in the O-point region of the islands where the electron temperature $\left(\mathrm{T}_{\mathrm{e}}\right)$ is nearly flat, and increased $\widetilde{n}$ at the X-point as well as radially outside of the island in line with the O-point, where gradients are large enough to linearly destabilize the Ion Temperature Gradient (ITG) and Trapped Electron Mode (TEM) turbulence. GENE non-linear gyrokinetic simulations of ion-scale $\widetilde{\mathrm{n}}$ across static magnetic islands can qualitatively replicate the observed scaling of $\widetilde{\mathrm{n}}$ with respect to the island width (W) inside and outside

\footnotetext{
a) Electronic mail: bardoczil@fusion.gat.com
}

of the radial range of $2 / 1$ NTM islands in DIII-D ${ }^{18,27}$.

In line with $\widetilde{n}$, turbulent electron temperature fluctuations $\left(\widetilde{\mathrm{T}}_{\mathrm{e}}\right)$ are also expected to drive transport across the island separatrices, supporting finite pressure gradient in a boundary layer inside the island separatrices and thereby impacting NTM stability. Measurements of $\widetilde{T}_{e}$ have been limited to non-rotating $2 / 1$ islands driven externally by resonant magnetic perturbation in $\mathrm{KSTAR}^{29}$ and to $1 / 1$ classical tearing driven islands in HL-2 $\mathrm{A}^{30}$. However, $\widetilde{T}_{e}$ measurements have not been reported for NTMs in reactor relevant plasma conditions. In both of the aforementioned experiments the Electron Cyclotron Emission (ECE) imaging diagnostic was utilized to probe $\mathrm{T}_{\mathrm{e}}$ in saturated islands.

Diagnostics based upon ECE are widely used for $T_{e}$ profile measurements and given sufficient spatiotemporal resolution, can be used to investigate turbulent $\widetilde{\mathrm{T}}_{\mathrm{e}}$. However, it is difficult to measure small $\widetilde{\mathrm{T}}_{\mathrm{e}}$ in H-mode plasmas, typically $\approx 1 \%$, via a standard ECE radiometer due to the inherent thermal noise in the ECE signals. A solution to this problem is offered by the Correlation ECE diagnostic (CECE), which is a two-channel ECE radiometer designed for $\widetilde{\mathrm{T}}_{\mathrm{e}}$ measurements. Crosscorrelation of two closely spaced ECE channels having uncorrelated thermal noise reveals small $\widetilde{\mathrm{T}}_{\mathrm{e}}$.

In this paper we report measurements of localized $\widetilde{T}_{e}$ across naturally rotating $2 / 1$ NTM magnetic islands in DIII-D ${ }^{31}$ high confinement mode (H-mode) plasmas via the $\mathrm{CECE}^{32}$ diagnostic along with qualitative comparison to $\mathrm{GENE}^{33}$ non-linear gyrokinetic simulations with magnetic islands. $\widetilde{\mathrm{T}}_{\mathrm{e}}$ measurements were taken in the saturated phase of the NTM as well as during the NTM 
growing phase. Note that the measurement of a $\widetilde{T}_{\mathrm{e}}$ fluctuation level requires typically at least a $200 \mathrm{~ms}$ time window but NTMs grow and saturate in typically about $100 \mathrm{~ms}$ after onset. In order to reconstruct the $\widetilde{\mathrm{T}}_{\mathrm{e}}(r)$ radial profile at a set of different island widths, the NTM growing phase was sub divided into a set of time windows, each characterized by a different average W. Next, the windows characterized by the same $\mathrm{W}$ in a series of repeat discharges were grouped together and $\widetilde{\mathrm{T}}_{\mathrm{e}}(r)$ was computed from each group of windows separately. By doing so, the scaling of the $\widetilde{\mathrm{T}}_{\mathrm{e}}(r)$ radial profile across the island is resolved with respect to $\mathrm{W}$. The reported measurements of turbulence properties and response to magnetic islands are important as they provide information to study NTM-turbulence interaction, as well as to test and validate turbulence and transport simulation codes.

Complementing the experimental data, we qualitatively compare the CECE data to GENE gyrokinetic simulations with magnetic islands. These simulations are run with more realistic island shapes than earlier studies ${ }^{27}$ to correctly account for the radial asymmetry of the perturbed flux, which is expected to impact flow shear and turbulence levels ${ }^{18,29} \cdot \widetilde{\mathrm{T}}_{\mathrm{e}}$ decreases (increases) with $\mathrm{W}$ inside (outside) of the radial range of the island when $\mathrm{W}$ is larger than a few times the ion gyroradius. These are in qualitative agreement with mesured $\widetilde{\mathrm{T}}_{\mathrm{e}}$ reported here and measured $\widetilde{\mathrm{n}}$ reported earlier ${ }^{25-27}$. The radial profile of $\widetilde{T}_{e}$ modification due to the island is asymmetric about the rational surface with smaller (larger) $\widetilde{T}_{e}$ in the region where larger (smaller) flow shear is expected due to the radially asymmetric island shape. Interestingly, CECE detects increased $\widetilde{\mathrm{T}}_{\mathrm{e}}$ near $q=2$ when $\mathrm{W}$ is a few times the ion gyroradius. This effect is captured by the gyrokinetic simulations, where the narrow island damps zonal flows and thereby causes an increase in turbulence in the X-point region. This mechanism may be related to $\widetilde{\mathrm{n}}$ increase due to radial magnetic fields driven externally by resonant magnetic perturbation during ELM suppression, an effect that was predicted theoretically ${ }^{34,35}$ and observed ${ }^{36}$ in DIII-D.

The paper is structured as follows. The experiments and diagnostics are described in Section II. Magnetic island effects on $\widetilde{T}_{\mathrm{e}}$ turbulence are described in Section III, followed by gyrokinetic simulations in Section IV. The paper is then concluded with a comparison of the DIII-D data and GENE simulation results in Section V.

\section{EXPERIMENTAL OVERVIEW}

We studied low- $k$ broadband $\widetilde{\mathrm{T}}_{\mathrm{e}}$ interaction with naturally growing and rotating $2 / 1 \mathrm{NTMs}$ in reproducible, stationary $\mathrm{H}$-mode plasma conditions with an 8 channel CECE diagnostic ${ }^{32}$ in DIII-D ${ }^{31}$. Growing magnetic islands flatten profiles in the O-point region and transiently increase gradients outside of the island region ${ }^{37}$. Possible concomitant transient changes in $\widetilde{\mathrm{T}}_{\mathrm{e}}$ can there- fore be studied across growing islands in non-equilibrium conditions, in contrast to saturated islands. $\widetilde{\mathrm{T}}_{\mathrm{e}}$ modifications by saturated islands has been reported earlier ${ }^{29,30}$.

An increase in turbulence outside of the island may contribute to the decrease of plasma stored energy in the NTM growing phase. This growing phase may also be used as a $\mathrm{W}$ scan by sub-dividing the transient into short time windows. We note that one may alternatively scan the saturated island width $\left(\mathrm{W}_{\mathrm{SAT}}\right)$ shot-to-shot and probe $\widetilde{T}_{e}$ in the flat-top phase of the discharges with fixed heat and particle sources. However, fluxes balance the fixed sources in equilibrium and gradients are not increased outside of the island, therefore a substantial increase of turbulence is not expected outside of the island in the flat-top phase ${ }^{25}$.

However, to resolve the small amplitude $\widetilde{\mathrm{T}}_{\mathrm{e}}$ level from thermal noise with the CECE diagnostic, more than 1000 sub data sets are required, corresponding to about $200 \mathrm{~ms}$ data with 2048 points in one FFT with $50 \%$ overlap. As NTMs saturate typically in about $100 \mathrm{~ms}$ after onset, the temporal resolution of the measured $\widetilde{T}_{e}$ is not high enough to investigate $\widetilde{T}_{e}$ evolution within the growing phase of a magnetic island in a single discharge, hence repeat discharges are required. The required data was collected in a series of 10 repeat discharges, where the NTM growing phase was then sub-divided into 5 time windows of at least $200 \mathrm{~ms}$, enabling the $\widetilde{\mathrm{T}}_{\mathrm{e}}(r)$ profile to determined at 5 different values of $\mathrm{W}$.

To ensure that local conditions for turbulence growth around the islands are comparable across the analyzed discharges, such as $n_{e}, T_{e}, T_{i}$ and their gradients, highly reproducible plasmas with similar magnetic island evolution and robust confinement response are required. These requirements were fulfilled by hybrid discharges with $m / n=2 / 1$ NTMs of $\mathrm{W}_{\mathrm{SAT}} \approx 11 \mathrm{~cm}$ at $\rho \approx 0.4$, rotating with $\mathrm{f}_{1} \approx 5 \mathrm{kHz}$. The major radius coordinate of the $q=m / n$ surface was $\mathrm{R}_{\mathrm{s}} \approx 203 \mathrm{~cm}$. Typical values of relevant plasma parameters: major radius of the magnetic axis was $\mathrm{R}=179 \mathrm{~cm}$, minor radius $\mathrm{a}=58 \mathrm{~cm}$, elongation was $\kappa=1.85$, plasma current $\mathrm{I}_{\mathrm{p}}=1 \mathrm{MA}$. $8 \mathrm{MW}$ neutral beam power was injected with a peakto-peak modulation amplitude of $6 \mathrm{MW}$. The electron temperature $\left(\mathrm{T}_{\mathrm{e}}\right.$, electron cyclotron emission, $\left.\mathrm{ECE}^{38}\right)$ and ion temperature $\left(\mathrm{T}_{\mathrm{i}}\right.$, charge exchange recombination, $\mathrm{CER}^{39}$ ) at $q=2$ were approximately $3.2 \mathrm{keV}$. In the stationary state the chord averaged electron density was $\mathrm{n}_{\mathrm{e}} \approx 3.8 \times 10^{19} \mathrm{~m}^{-3}$ (interferometry ${ }^{40}$ ), the toroidal magnetic field was $\mathrm{B}_{\mathrm{T}}=2.05 \mathrm{~T}$ in the counter- $\mathrm{I}_{\mathrm{p}}$ direction, normalized plasma beta was $\beta_{\mathrm{N}}=2\left(\beta a \mathrm{~B}_{\mathrm{T}} / \mathrm{I}_{\mathrm{P}}\right)$.

NTM evolution and the confinement response are similar across these plasmas as seen from the time traces of $\mathrm{W}, \mathrm{T}_{\mathrm{e}}$ and $\mathrm{n}_{\mathrm{e}}$ in Fig. 1 (a-c), where time is measured relative to the NTM onset time $\left(t_{\circ}\right)$ for convenience. Similarity between NTM dynamics and confinement response across discharges is quantified by the relationship between the standard deviation $(\bar{\sigma})$ of the time average of $\mathrm{T}_{\mathrm{e}}, \mathrm{n}_{\mathrm{e}}$ and the $n=1$ Mirnov amplitude $\left(\mathrm{B}_{1}\right)$ and fre- 

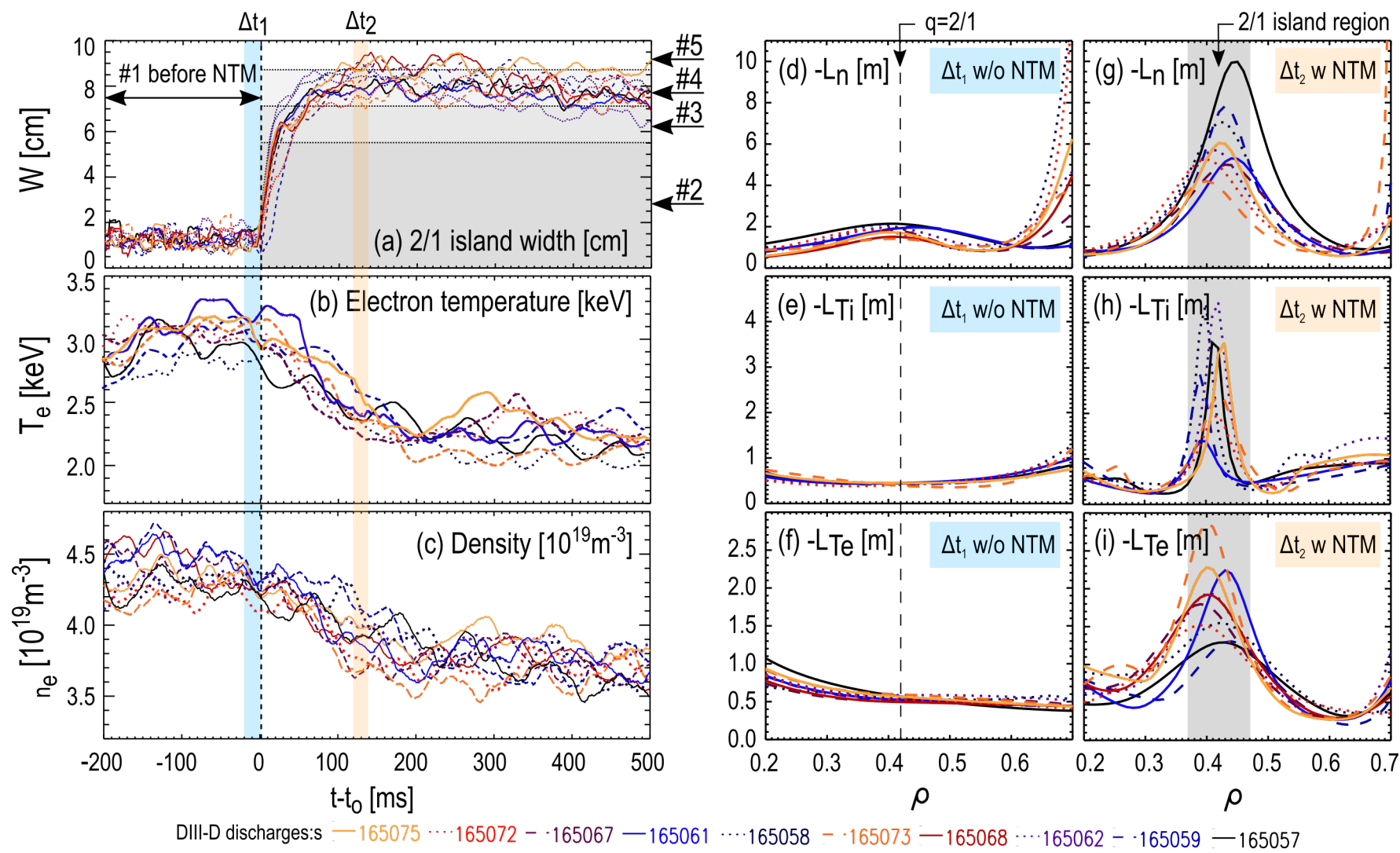

FIG. 1: Time history of the (a) island width, (b) ECE electron temperature at $q=2$ and (c) line integrated density. Rotation averaged negative scale lengths before NTM onset (w/o NTM) (d) $-\mathrm{L}_{\mathrm{n}},(\mathrm{e})-\mathrm{L}_{\mathrm{Ti}},(\mathrm{f})-\mathrm{L}_{\mathrm{Te}}$ and after NTM saturation $\left(\mathrm{w}\right.$ NTM) (g) $-\mathrm{L}_{\mathrm{n}},(\mathrm{h})$

$-\mathrm{L}_{\mathrm{Ti}},(\mathrm{i})-\mathrm{L}_{\mathrm{Te}}$. These negative scale lengths were calculated from cubic splines fitted to the measured profiles of $n_{e}, T_{i}$ and $T_{e}$.

quency $\left(\mathrm{f}_{1}\right)$ within shots and the ensemble averaged standard deviation within shots $(\sigma) . \bar{\sigma}$ and $\sigma$ are compared in a window before NTM onset $\left(\Delta t_{1}=\left(t_{1}, t_{1}+\Delta t\right)\right)$ and after NTM saturation $\left(\Delta t_{2}=\left(t_{2}, t_{2}+\Delta t\right)\right) . \Delta t=20 \mathrm{~ms}$ windows were used starting $t_{1}=20 \mathrm{~ms}$ before NTM onset and $t_{2}=120 \mathrm{~ms}$ after NTM onset in the saturated state. These time windows are highlighted in Fig. 1 (a-c). $t_{1}$ and $t_{2}$ were chosen to ensure the data is taken from stationary phases close to the transient. The $\sigma$ and $\bar{\sigma}$ metrics are defined as follows. $\mathrm{M}_{b, i}^{\mathrm{T}_{\mathrm{e}}}$ is the time average of $\mathrm{T}_{\mathrm{e}}$ within the $i^{\text {th }}$ discharge in the $\Delta t_{1}$ time window and $\mathrm{M}_{b}^{\mathrm{T}_{\mathrm{e}}}$ is the ensemble average of $\mathrm{M}_{b, i}^{\mathrm{T}_{\mathrm{e}}}$ over all discharges:

$$
\mathrm{M}_{b, i}^{\mathrm{T}_{\mathrm{e}}}=\frac{1}{\Delta t} \sum_{k=1}^{n} \mathrm{~T}_{\mathrm{e}}\left(t_{k}\right), \quad \mathrm{M}_{b}^{\mathrm{T}_{\mathrm{e}}}=\frac{1}{N} \sum_{i=1}^{N} \mathrm{M}_{b, i}^{\mathrm{T}_{\mathrm{e}}},
$$

where the summation over $k$ is carried out in the $\Delta t_{1}$ interval. The standard deviation of $\mathrm{M}_{b, i}^{\mathrm{T}_{\mathrm{e}}}$ across all discharges is:

$$
\bar{\sigma}_{b}^{\mathrm{T}_{\mathrm{e}}}=\sqrt{\frac{\sum_{i=1}^{N}\left(\mathrm{M}_{b}^{\mathrm{T}_{\mathrm{e}}}-\mathrm{M}_{b, i}^{\mathrm{T}_{\mathrm{e}}}\right)^{2}}{N-1}} .
$$

The ensemble averaged standard deviation is:

$$
\sigma_{b}^{\mathrm{T}_{\mathrm{e}}}=\frac{1}{N} \sum_{i=1}^{N} \sqrt{\frac{\sum_{k=1}^{n}\left(\mathrm{M}_{b, i}^{\mathrm{T}_{\mathrm{e}}}-\mathrm{T}_{\mathrm{e}}\left(t_{k}\right)\right)^{2}}{n-1}},
$$

Here $N$ is the number of discharges, $n$ is the number of samples within a shot in the $\Delta t_{1}$ interval and the index $b$ stands for "before NTM onset". The same definitions hold for the $\Delta t_{2}$ interval, where the index $a$ stands for "after NTM saturation". The same definitions are used for the $n_{e}, B_{1}$ and $f_{1}$ quantities and numerical values are summarized in Table I.

\begin{tabular}{|l|c|c|c|c|}
\hline Before NTM onset & $\mathrm{M}_{b}$ & $\bar{\sigma}_{b}$ & $\bar{\sigma}_{b}[\%]$ & $\sigma_{b}$ \\
\hline $\mathrm{T}_{\mathrm{e}}[\mathrm{keV}]$ & 3.2 & 0.18 & 5.6 & 0.10 \\
$\mathrm{n}_{\mathrm{e}}\left[10^{19} \mathrm{~m}^{-3}\right]$ & 4.4 & 0.11 & 2.7 & 0.09 \\
$\mathrm{~B}_{1}\left[10^{-4} \mathrm{~T}\right]$ & 1.2 & 0.16 & 13.3 & 0.29 \\
\hline \hline After saturation & $\mathrm{M}_{a}$ & $\bar{\sigma}_{a}$ & $\bar{\sigma}_{a}[\%]$ & $\sigma_{a}$ \\
\hline $\mathrm{T}_{\mathrm{e}}[\mathrm{keV}]$ & 2.3 & 0.17 & 7.4 & 0.08 \\
$\mathrm{n}_{\mathrm{e}}\left[10^{19} \mathrm{~m}^{-3}\right]$ & 3.8 & 0.09 & 2.3 & 0.09 \\
$\mathrm{~B}_{1}\left[10^{-4} \mathrm{~T}\right]$ & 7.8 & 0.52 & 6.7 & 0.30 \\
$\mathrm{f}_{1}[\mathrm{kHz}]$ & 5.4 & 0.45 & 8.4 & 0.32 \\
\hline
\end{tabular}

TABLE I: Comparison of $\mathrm{T}_{\mathrm{e}}, \mathrm{n}_{\mathrm{e}}, \mathrm{n}=1$ Mirnov amplitude and frequency across all discharges before NTM onset \& in the saturated phase of the NTM.

Variations of $\mathrm{T}_{\mathrm{e}}, \mathrm{n}_{\mathrm{e}}$ and $\mathrm{B}_{1}$ across the discharges $\left(\bar{\sigma}_{b}\right)$ are comparable to the corresponding variations within shots $\left(\sigma_{b}\right)$. Therefore, we use these discharges to derive average $\widetilde{T}_{e}$ levels within time windows where $\mathrm{W}$ falls in one of the five pre-defined ranges listed in Table II. These 
five ranges of $W$ were selected in each discharge such that even the shortest window (the one right after onset where the NTM growth is the fastest) integrates to at least $200 \mathrm{~ms}$ over the set of discharges. These ranges (\#1 - \#5) are also highlighted in Fig. 1 (a).

\begin{tabular}{|l|c|c|c|}
\hline window & $\mathrm{W}_{\min }[\mathrm{cm}]$ & $\mathrm{W}_{\max }[\mathrm{cm}]$ & $\mathrm{W}_{\text {mean }}[\mathrm{cm}]$ \\
\hline$\# 1$ (ref.) & no island & N/A & N/A \\
$\# 2$ & 0 & 5.50 & 2.8 \\
$\# 3$ & 5.50 & 7.10 & 6.3 \\
$\# 4$ & 7.10 & 8.75 & 7.9 \\
$\# 5$ & 8.75 & 10.0 & 9.4 \\
\hline
\end{tabular}

TABLE II: Time window selection criteria for each discharge. Window \#1 is used as a reference window w/o NTM. Windows \#2-5 represent phases during NTM evolution with different W.

Experimental characterization of the island structure is discussed in Subsection II A. NTM impact on plasma profiles and expected linear effects on micro-turbulence stability at $q=2$ are described in Subsection IIB.

\section{A. Measurement of the Island Width (ECE and Mirnov)}

The structure, location, rotation and width of the $2 / 1$ islands were monitored via the DIII-D ECE radiometer which measures high resolution $\mathrm{T}_{\mathrm{e}}$ from optically thick, second harmonic (X-mode) electron cyclotron emission. $\mathrm{T}_{\mathrm{e}}$ was probed at 40 radial locations with $480 \mathrm{kHz}$ sampling rate $0.3 \mathrm{~cm}$ above the tokamak mid-plane. Island rotation enables $T_{\mathrm{e}}$ to be probed with respect to the island helical phase $\xi=m \theta-n \phi$. In the lab frame $\xi=\omega_{\circ} t$, where $\omega_{\circ} /(2 \pi)$ is the island frequency, and $t$ is time. The perturbed flux surfaces of the island are labeled by $\Omega$ :

$$
\Omega=8 X^{2}+(2 \mathrm{~A} X+1) \cos (\xi)
$$

Here $X=\left(R-R_{\mathrm{s}}\right) / \mathrm{W}$ and $\mathrm{A}$ is the dimensionless radial asymmetry parameter ${ }^{41} . \Omega=1-\mathrm{A}^{2} / 8$ maps out the separatrix of the island. To characterize the islands, the $\mathrm{W}$, $\mathrm{R}_{\mathrm{s}}$ and $\mathrm{A}$ parameters were determined by fitting the solutions of an anisotropic heat transport model of magnetic islands ${ }^{42}$ to the ECE data in $5 \mathrm{~ms}$ windows. This method solves the $\nabla_{\perp}^{2} \chi_{\perp} \mathrm{T}_{\mathrm{e}}+\nabla_{\|}^{2} \chi_{\|} \mathrm{T}_{\mathrm{e}}=0$ transport equation in the island geometry in a grid of the parameter space defined by $\mathrm{W}, \mathrm{R}_{\mathrm{s}}, \chi_{\perp} / \chi_{\|}$and $\mathrm{A}$. Here $\chi_{\perp}$ and $\chi_{\|}$are the perpendicular and parallel electron thermal diffusivities, respectively. The coordinates of the grid point where the model produces the smallest squared error, compared to the experimental data, are the best estimators of the island parameters. Typical values are $\mathrm{W} \approx 5-10 \mathrm{~cm}$, $\chi_{\perp} / \chi_{\|}=10^{-8}$ and $\mathrm{A}=-0.6$.

This method is applicable when $\mathrm{W}$ is larger than the radial resolution of the ECE measurement and when the island is at least partially flattened, which are satisfied typically when $\mathrm{W}>5 \mathrm{~cm}$. The island width determination was extended to the $\mathrm{W}<5 \mathrm{~cm}$ by combining the above described method with external magnetic mea- surements as, for example, in integrated TRANSP simulations of NTM driven fast ion transport ${ }^{43}$.

\section{B. Plasma Profiles and Linearly Unstable Drift Waves}

Determining the type of unstable drift-waves across the island requires nonlinear gyrokinetic simulations with magnetic islands and plasma parameters $\left(\mathrm{T}_{\mathrm{e}}, \mathrm{T}_{\mathrm{i}}, \nabla \mathrm{T}_{\mathrm{e}}\right.$, $\nabla \mathrm{T}_{\mathrm{i}}, \nabla \mathrm{n}_{\mathrm{e}}$, geometry, rotation, etc.) matched to the experiment, which is beyond the scope of this paper. However, to identify the type of dominant micro-instability of the background plasma at $q=2$ before NTM onset, in the $k$-range probed by the CECE diagnostic, we used linear GENE gyro-kinetic simulations ${ }^{33}$ with the experimental profiles measured before NTM onset as in earlier work $^{26-28}$. Typically, the most unstable mode of these plasmas around $\rho \approx 0.4$ is the ITG instability and $\mathrm{L}_{\mathrm{n}} / \mathrm{L}_{\mathrm{Ti}}$ and $T_{e} / T_{i}$ are good indicators for the stability of this mode ${ }^{44-46}$. At higher values of $\mathrm{L}_{\mathrm{n}} / \mathrm{L}_{\mathrm{Ti}}$ and $\mathrm{T}_{\mathrm{e}} / \mathrm{T}_{\mathrm{i}}$ ITG modes are more unstable.

Measured profiles of $\mathrm{n}_{\mathrm{e}}$ (Thomson scattering ${ }^{47}$ ), $\mathrm{T}_{\mathrm{e}}$ (ECE and Thomson scattering) and $\mathrm{T}_{\mathrm{i}}$ (Charge Exchange Recombination ${ }^{39}$ ) were used to calculate the neg-

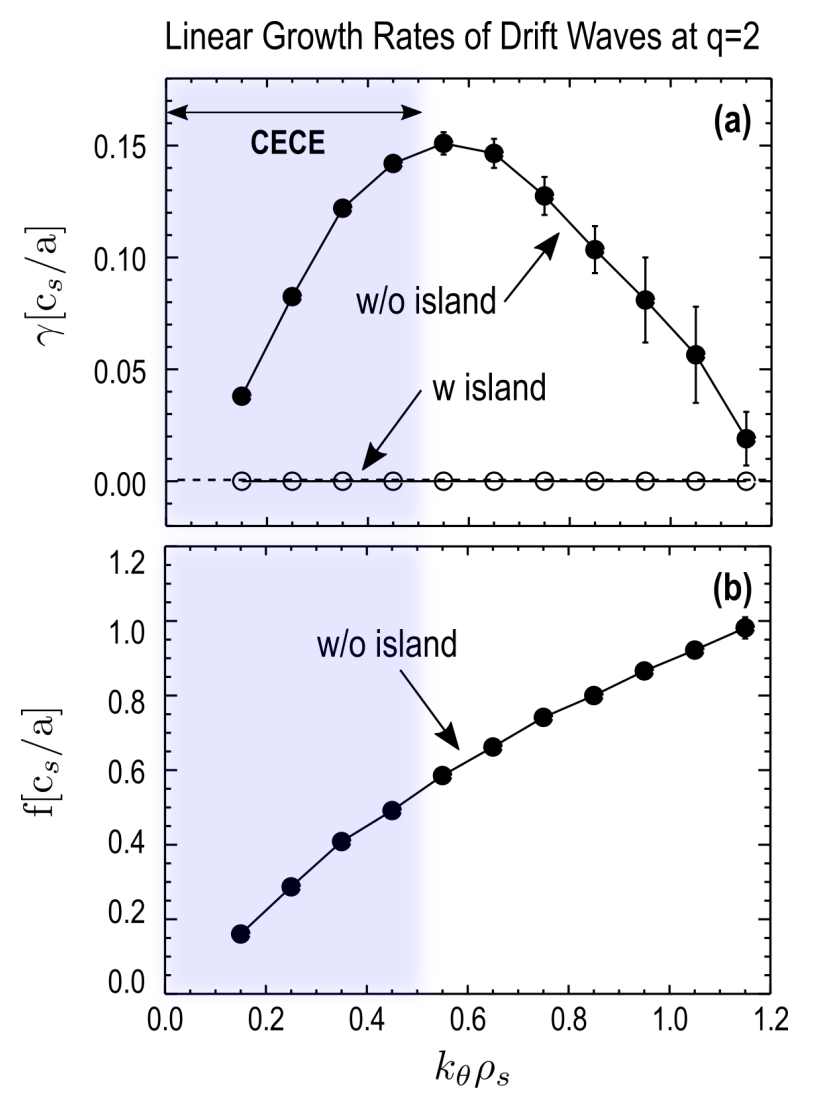

FIG. 2: (a) Linear growth rates and (b) frequencies of unstable drift waves at $q=2$ before NTM onset (filled circles) and after NTM saturation (open circles) via GENE (see input experimental profiles in Fig. 1). Shaded column marks the sensitivity range of the $\widetilde{\mathrm{T}}_{\mathrm{e}}$ turbulence diagnostic (CECE). 
ative gradient length-scales $-\mathrm{L}_{\mathrm{n}}=-\mathrm{n}_{\mathrm{e}} / \nabla \mathrm{n}_{\mathrm{e}},-\mathrm{L}_{\mathrm{Ti}}=$ $-\mathrm{T}_{\mathrm{i}} / \nabla \mathrm{T}_{\mathrm{i}}$ and $-\mathrm{L}_{\mathrm{Te}}=\mathrm{T}_{\mathrm{e}} / \nabla \mathrm{T}_{\mathrm{e}}$ in the $\Delta t_{1}$ time window before NTM onset [Fig. 1 (d-f)] and in the $\Delta t_{2}$ time window after NTM onset in the saturated state [Fig. 1 (g-i)].

These negative gradient length-scale profiles are flat without an island at $q=2$ [Fig. 1 (d-f)] but peaked inside the $2 / 1$ island region after NTM saturation [Fig. 1 (g-i)]. Before NTM onset $\mathrm{L}_{\mathrm{n}} / \mathrm{L}_{\mathrm{Ti}}=4.15 \pm 0.40$ and $\mathrm{T}_{\mathrm{i}} / \mathrm{T}_{\mathrm{e}}=$ $1.17 \pm 0.06$ in the analyzed 10 discharges but after NTM saturation $\mathrm{L}_{\mathrm{n}} / \mathrm{L}_{\mathrm{Ti}}=1.86 \pm 0.44$ and $\mathrm{T}_{\mathrm{i}} / \mathrm{T}_{\mathrm{e}}=1.01 \pm 0.04$. The decrease of $\mathrm{L}_{\mathrm{n}} / \mathrm{L}_{\mathrm{Ti}}$ by about a factor of 2 is a strong indication that ITG modes are linearly more stable inside the island region than in the background plasma at $q=2$ before NTM onset in all of these plasmas.

Our GENE flux-tube simulations were run on ionscales $\left(k_{\theta} \rho_{s}=0.1-1.2\right)$, used two fully kinetic species (deuterons and electrons) and included electromagnetic effects. Here $k_{\theta}$ is the poloidal wavenumber and $\rho_{s}=$ $\mathrm{c}_{s} / \omega_{i} \approx 0.4 \mathrm{~cm}$, where $\mathrm{c}_{s}=\sqrt{\mathrm{T}_{\mathrm{e}} / \mathrm{m}_{i}} \approx 338 \mathrm{~km} / \mathrm{s}$, and $\mathrm{m}_{i}$ and $\omega_{i}$ are the ion mass and cyclotron frequency, respectively. A dominant mode in the range of the Ion Temperature Gradient (ITG) instability that propagates in the ion diamagnetic direction was found at long wavelengths $\left(k_{\theta} \rho_{s} \lesssim 1.2\right)$ before NTM onset [Fig. 2]. The growth rate $(\gamma)$ of this mode increases with $\mathrm{T}_{\mathrm{i}} / \mathrm{T}_{\mathrm{e}}$ and with $\mathrm{L}_{\mathrm{n}} / \mathrm{L}_{\mathrm{Ti}}$, which are good indicators of the ITG instability, as mentioned before. Variations of $\gamma$ across discharges are due variations of profile gradients. Collision frequencies were small and varied slightly and did not affect $\gamma$ significantly. When using the profiles from after NTM saturation [Fig. 1 (g-i)], all modes become stable. This suggest that all modes are linearly stable at the O-point of the islands. As the CECE diagnostic is sensitive in the $k_{\theta} \rho_{s}<0.5$ range, the probed fluctuations discussed in Section III are presumably due to ITG turbulence.

\section{TURBULENT $\widetilde{T}_{\mathrm{e}}$ CHARACTERISTICS AND RESPONSE TO NTMS (CECE)}

Correlation ECE (CECE) diagnostic was used to investigate the interaction between low- $k \widetilde{\mathrm{T}}_{\mathrm{e}}$ with NTM in this study. CECE is a diagnostic technique to measure $\widetilde{\mathrm{T}}_{\mathrm{e}}$ masked by thermal noise in the ECE radiometer signal through a cross correlation technique ${ }^{48}$. The CECE diagnostic at DIII-D uses $2^{\text {nd }}$ harmonic X-mode ECE radiation and removes thermal noise through a spectral decorrelation scheme, correlating two ECE channels in the disjoint frequency band but located within the correlation length of $\widetilde{T}_{e}{ }^{49}$. This CECE system ${ }^{32}$ was probing $\widetilde{\mathrm{T}}_{\mathrm{e}}$ at 8 radial locations simultaneously inside and outside of the radial range of the magnetic islands in the outboard mid-plane. CECE data shown in this study are from optically thick plasma (optical depth, $\tau>20$ ), so the effect of $\widetilde{\mathrm{n}}$ on the CECE measurements ${ }^{50}$ is negligible here. Non-thermal emission due to MHD modes such as ELM ${ }^{51}$ was also excluded in this analysis. Fig. 3

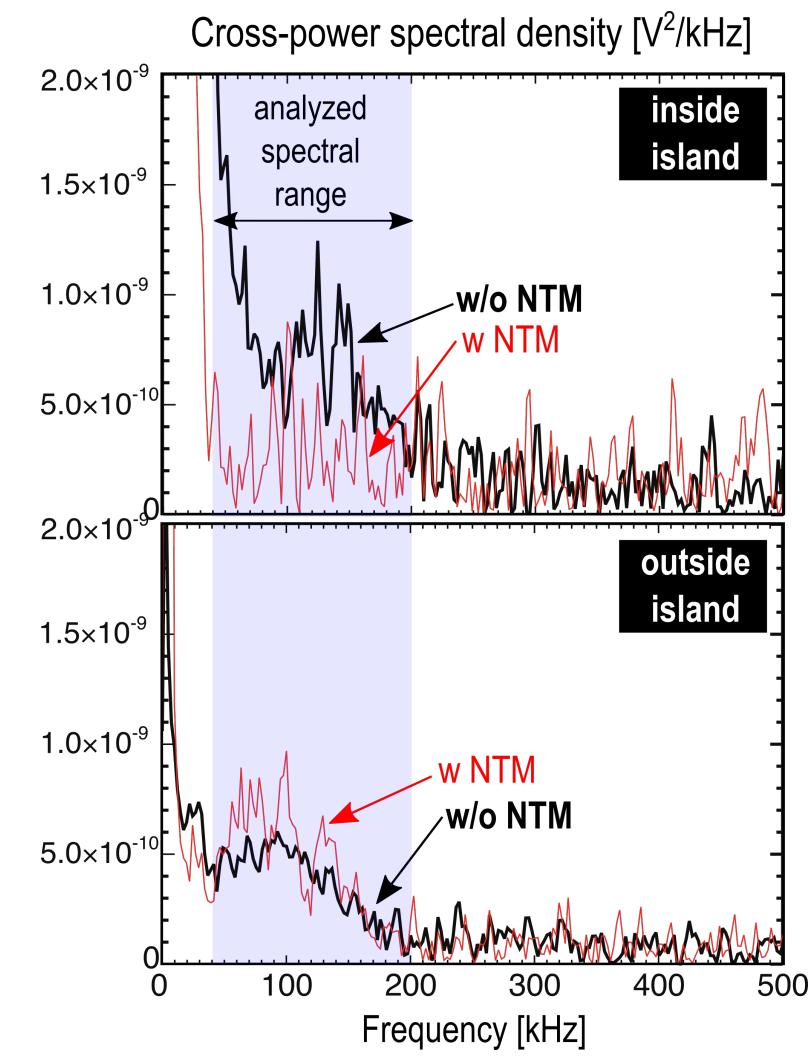

FIG. 3: CPSD of the CECE channels (a) radially inside and (b) radially outside of the island region. Thick (black) line indicates

$\widetilde{\mathrm{T}}_{\mathrm{e}}$ without NTM and thin (red) line indicates $\widetilde{\mathrm{T}}_{\mathrm{e}}$ with NTM

shows examples of the CECE cross power spectral density (CPSD) inside and outside of the radial range of the island. Broadband fluctuations in the $\Delta f=50-200 \mathrm{kHz}$ range are attributed to $\mathrm{T}_{\mathrm{e}}$ turbulence $\left(\widetilde{\mathrm{T}}_{\mathrm{e}}\right)$. Fig. 3 (a) shows that within the island region $\widetilde{\mathrm{T}}_{\mathrm{e}}$ is smaller with NTM compared to before NTM onset, while Fig. 3 (b) shows that radially outside of the island region $\widetilde{\mathrm{T}}_{\mathrm{e}}$ is larger with NTM compared to before NTM onset.

These broadband fluctuations shown in Fig. 3 are presumably due to ITG turbulence, as discussed in Subsection II B. The integral of the CPSD over the $\Delta f$ range is then used to monitor changes of $\widetilde{\mathrm{T}}_{\mathrm{e}}$ inside and outside of the radial range of the island during NTM evolution. Note, that this $\widetilde{\mathrm{T}}_{\mathrm{e}}$ is rotation averaged and the helical structure is not resolved (e.g. differences between $\mathrm{X}$-point and O-point fluctuation levels are not obtained). Resolution vs. $\xi$ would require even more data samples and therefore additional sets of repeat discharges. The change of the $\widetilde{\mathrm{T}}_{\mathrm{e}}(R)$ profile caused by the island is calculated as:

$$
\Delta \widetilde{\mathrm{T}}_{\mathrm{e}}(R)=\left\langle\frac{\widetilde{\mathrm{T}}_{\mathrm{e}}(R)-\widetilde{\mathrm{T}}_{\mathrm{e}, \mathrm{W}=0}(R)}{\widetilde{\mathrm{T}}_{\mathrm{e}, \mathrm{W}=0}(R)}\right\rangle_{\xi},
$$

where $\widetilde{\mathrm{T}}_{\mathrm{e}, \mathrm{W}=0}(R)$ is the radial profile of the fluctuations before NTM onset. The four profiles in Fig. $4(\mathrm{i}, \mathrm{j}, \mathrm{k}, \mathrm{l})$ 


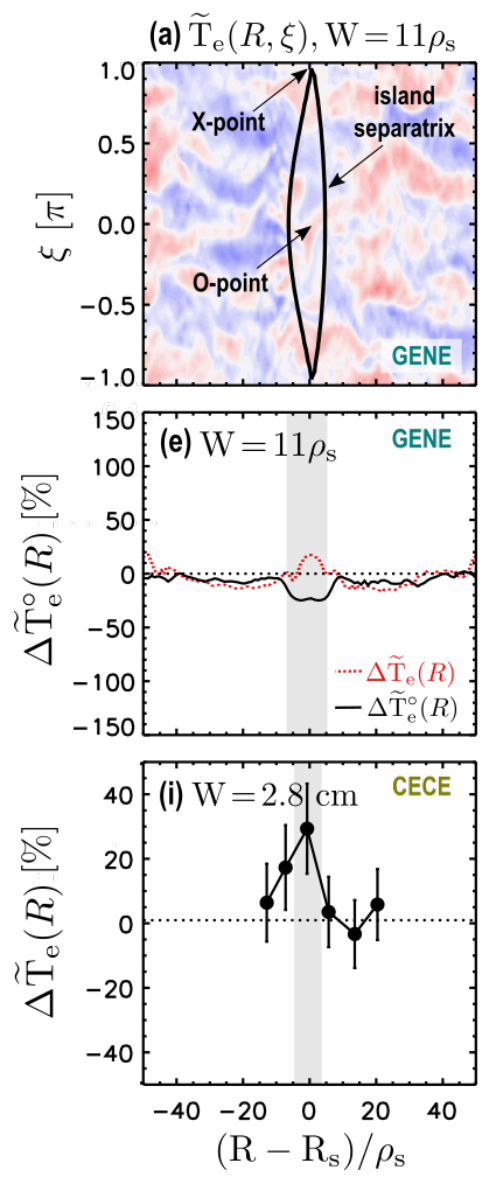

(b) $\widetilde{\mathrm{T}}_{\mathrm{e}}(R, \xi), \mathrm{W}=22 \rho_{\mathrm{s}}$
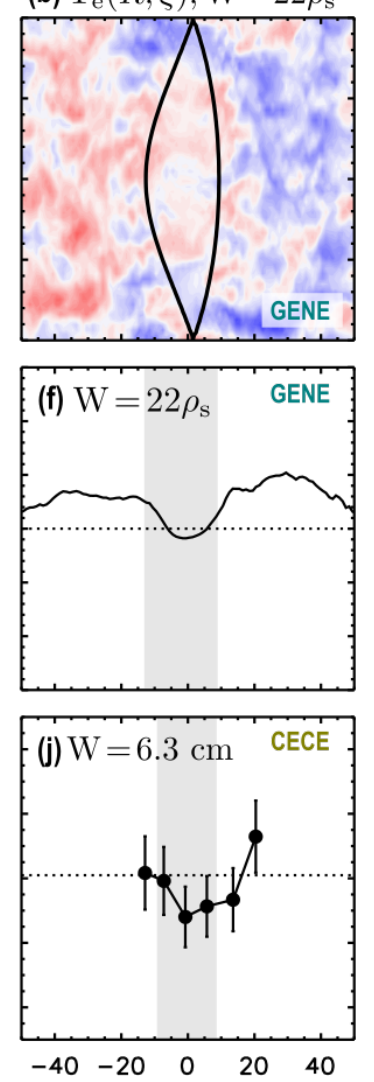

$\left(\mathrm{R}-\mathrm{R}_{\mathrm{s}}\right) / \rho_{\mathrm{s}}$ (c) $\widetilde{\mathrm{T}}_{\mathrm{e}}(R, \xi), \mathrm{W}=33 \rho_{\mathrm{s}}$
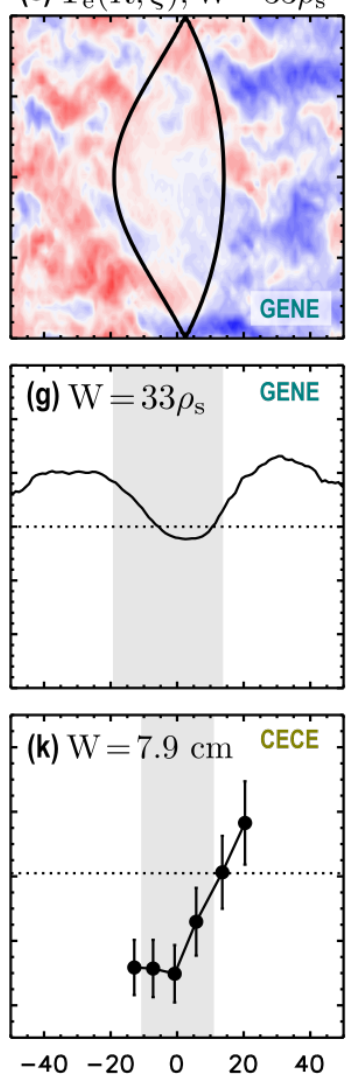

$\left(\mathrm{R}-\mathrm{R}_{\mathrm{s}}\right) / \rho_{\mathrm{s}}$
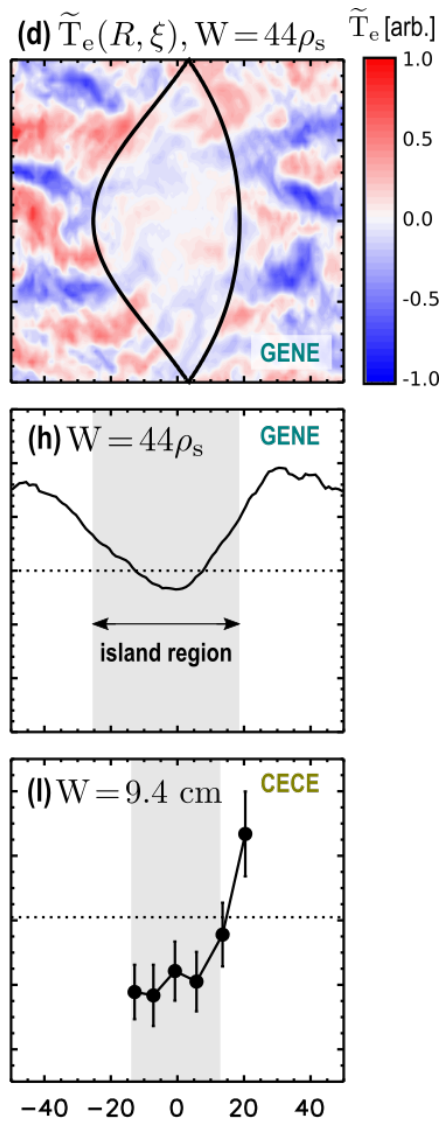

$\left(\mathrm{R}-\mathrm{R}_{\mathrm{s}}\right) / \rho_{\mathrm{s}}$

FIG. 4: (a-d) Contour of instantaneous $\widetilde{\mathrm{T}}_{\mathrm{e}}$ in GENE simulations. (e-h) Radial profiles of $\widetilde{\mathrm{T}}_{\mathrm{e}}$ changes due to an island in GENE simulations. (i-l) Radial profiles of CPSD of CECE channels.

show $\Delta \widetilde{\mathrm{T}}_{\mathrm{e}}(R)$ right after island formation [Fig. 4 (i)], at two stages of the island growth [Fig. $4(\mathrm{j}, \mathrm{k})]$ and in the saturated state [Fig. 4 (l)]. When a narrow island is formed $(\mathrm{W}=2.8 \mathrm{~cm})$, the probed $\widetilde{\mathrm{T}}_{\mathrm{e}}$ at $\mathrm{R}_{\mathrm{s}}$ is higher than before island formation [Fig. 4 (i)]. As the island grows, $\widetilde{\mathrm{T}}_{\mathrm{e}}$ gradually decreases inside the island and increases outside of the island in the probed radial range. These changes are qualitatively expected based on the evolution of local gradients in these discharges which were shown earlier in Fig. 1. The maximum detected increase (reduction) are $30 \%(-30 \%)$. These changes in $\widetilde{\mathrm{T}}_{\mathrm{e}}$ during NTM growth are in qualitative agreement with the changes in $\widetilde{\mathrm{n}}$ in the 165064 DIII-D plasma ${ }^{27}$.

A radial asymmetry of $\widetilde{\mathrm{T}}_{\mathrm{e}}$ is also evident in [Fig. $4(\mathrm{j})$, (k), (l)]. Fluctuations are lower near the separatrix on the plasma core side of the island $\left(\mathrm{R}_{-}\right)$compared to near the separatrix on the plasma edge side of the island $\left(\mathrm{R}_{+}\right)$, where

$$
\mathrm{R}_{ \pm}=\mathrm{R}_{s} \pm \frac{\mathrm{W}}{2}\left(1 \pm \frac{\mathrm{A}}{4}+\frac{1}{2}\left(\frac{\mathrm{A}}{4}\right)^{2}\right)
$$

These results are important as an increase of $\widetilde{T}_{e}$ radially outside of the island may increase the thermal trans- port which is consistent with observed loss of thermal confinement due to $\mathrm{NTMs}^{1}$. On the other hand, reduced $\widetilde{\mathrm{T}}_{\mathrm{e}}$ at the O-point offers an explanation for reduced thermal transport at the O-point ${ }^{42,52,53}$ which is expected to have a destabilizing effect on the $\mathrm{NTM}^{25}$.

In Section IV, the above reported measurements will be compared to GENE non-linear gyrokinetic turbulence simulations with magnetic islands.

\section{IV. $\widetilde{T}_{\mathrm{e}}$ IN NON-LINEAR GYROKINETIC SIMULATIONS WITH MAGNETIC ISLANDS}

We have used GENE nonlinear gyrokinetic simulations in 3-dimensional, toroidal geometry with static magnetic islands to study magnetic island effects on ion-scale turbulence $^{18}$. Comparison to DIII-D BES data reported that the simulations with radially symmetric structure qualitatively replicate the observed scaling of $\widetilde{n}$ modification with island size ${ }^{27}$. However, for a more detailed comparison, here we also consider the effect of the radial asymmetry of the island. When $\mathrm{A}<0$, shear flows increase (decrease) at the island separatrix in line with the O-point near $R_{-}\left(R_{+}\right)$. This modified flow shear 
then feeds back to decrease (increase) local fluctuation levels ${ }^{18}$. As the islands are characterized by $\mathrm{A}=-0.6$, low (high) flow shear and increased (decreased) $\widetilde{\mathrm{T}}_{\mathrm{e}}$ is expected radially outside of the island near $R_{+}\left(R_{-}\right)$.

GENE self-consistently solves the gyrokinetic-Maxwell system of equations on a fixed grid in five dimensional phase space (plus time). Two particle species (deuterons and electrons) were used including electromagnetic effects. The equilibrium magnetic configuration (large aspect-ratio, circular model equilibrium) is characterized by a safety factor of $q=1.5$, magnetic shear of $\hat{s}=0.16$ and inverse aspect ratio of $\epsilon=0.19, \mathrm{R} / \mathrm{L}_{\mathrm{Ti}}=\mathrm{R} / \mathrm{L}_{\mathrm{Te}}=$ $6.9, \mathrm{R} / \mathrm{L}_{\mathrm{n}}=2.2$. These parameters are similar to the "Cyclone DIII-D base case parameter set" 54 (discharge \#81499) as in other gyrokinetic simulations ${ }^{13,55}$. As such, these simulations can only be compared qualitatively to the DIII-D experiments of this paper and the simulation results are presented in arbitrary units.

The simulations are started out with constant gradients which drive the ITG modes unstable. Next, the turbulent cross-field transport and the fast parallel transport inside the island lead to the modification of the initially imposed profile such that gradients are reduced at the O-point but increased outside the island region. Modified gradients in turn can lead to turbulence modification in a self-consistent way. The simulation is run much longer than the turbulence correlation time beyond which the system has come to statistical equilibrium. Note, that the equilibrium profiles are fixed through the boundary conditions, as the characteristic global confinement timescale of H-mode plasmas is much longer than the timescale of these simulations. However, the profiles quickly modify inside and around the island due to the parallel transport which has a much shorter time-scale than the global confinement.

The gyrokinetic turbulent electron temperature fluctuation amplitude $\widetilde{\mathrm{T}}_{\mathrm{e}}(R, \xi)$ is calculated as the root mean square of the instantaneous turbulent electron temperature fluctuations $\widetilde{\mathrm{T}}_{\mathrm{e}}(R, \xi, t)$ of the simulation output:

$$
\widetilde{\mathrm{T}}_{\mathrm{e}}(R, \xi)=\left(\left\langle\left(\widetilde{\mathrm{T}}_{\mathrm{e}}(R, \xi, t)-\left\langle\widetilde{\mathrm{T}}_{\mathrm{e}}(R, \xi, t)\right\rangle_{t}\right)^{2}\right\rangle_{t}\right)^{1 / 2}
$$

Snapshots of $\widetilde{\mathrm{T}}_{\mathrm{e}}(R, \xi, t)$ in Fig. 4 (a-d) show that instantaneous turbulent electron temperature fluctuations are slightly reduced inside but not affected substantially outside a small island $\left(\mathrm{W} \approx 11 \rho_{\mathrm{i}}\right)$. In contrast, $\widetilde{\mathrm{T}}_{\mathrm{e}}$ decreases (increases) inside (outside) large islands $\left(\mathrm{W}>11 \rho_{\mathrm{i}}\right)$. This is more clear from the radial profile of the relative change of the fluctuation level $\left(\Delta \widetilde{\mathrm{T}}_{\mathrm{e}}\right)$ averaged over the helical coordinate [Fig. 4 (e-h)], defined as:

$$
\Delta \widetilde{\mathrm{T}}_{\mathrm{e}}^{\circ}(R)=\frac{\left\langle\widetilde{\mathrm{T}}_{\mathrm{e}}(R, \xi)\right\rangle_{\xi}-\widetilde{\mathrm{T}}_{\mathrm{e}}^{\mathrm{ref}}}{\widetilde{\mathrm{T}}_{\mathrm{e}}^{\mathrm{ref}}}
$$

where $\widetilde{\mathrm{T}}_{\mathrm{e}}^{\mathrm{ref}}=\left\langle\widetilde{\mathrm{T}}_{\mathrm{e}, \mathrm{W}=0}\left(R_{\mathrm{B}}, \xi\right)\right\rangle_{\xi}$ is a constant reference fluctuation level, calculated at the boundary $\left(R_{\mathrm{B}}\right)$ of the simulation box without island. $\Delta \widetilde{\mathrm{T}}_{\mathrm{e}}(R)$ increases by maximum about $100 \%$ radially outside of the island region when $\mathrm{W} \approx 44 \rho_{s}$, and reduces by about $20 \%$ radially inside the island region. These changes are in qualitative agreement with increased (reduced) local gradients outside (inside) the island region.

Note that $\Delta \widetilde{\mathrm{T}}_{\mathrm{e}}^{\circ}(R)$ is radially asymmetric, as expected, due to the radially asymmetric island shape. With $\mathrm{A}=$ $-0.6, \Delta \widetilde{\mathrm{T}}_{\mathrm{e}}^{\circ}(R)$ is smaller at $R<\mathrm{R}_{-}$compared to $\Delta \widetilde{\mathrm{T}}_{\mathrm{e}}$ at $\mathrm{R}_{+}<R$.

Interestingly, when $\mathrm{W}$ is comparable to the radial correlation length of zonal flows (ZFs) at $q=m / n\left(\Delta \mathrm{R}_{\mathrm{zf}}\right)$, the perturbed magnetic geometry of the island reduces ZF growth rates and allows $\widetilde{\mathrm{T}}_{\mathrm{e}}$ saturation at a relatively higher level, see Subsection IV A.

\section{A. Effect of Narrow Islands on ZFs and $\widetilde{T}_{\mathrm{e}}$ Saturation}

ZFs have relatively large growth rates at $q=m / n$, which reduces local $\widetilde{\mathrm{T}}_{\mathrm{e}}$ levels in a narrow band of $\Delta \mathrm{R}_{\mathrm{zf}} \approx$ $10 \rho_{\mathrm{s}}$ around the rational surface in the simulation without islands [Fig. 5 (a)]. Interestingly, when $\mathrm{W} \geq \Delta \mathrm{R}_{\mathrm{zf}}$, ZFs are significantly suppressed which then allows $\widetilde{\mathrm{T}}_{\mathrm{e}}$ saturation at a higher level in the X-point region where gradients are not affected by the island [Fig. 5 (b)]. In turn, the local fluctuation level $\left(\Delta \widetilde{\mathrm{T}}_{\mathrm{e}}(R)\right)$ increases at the rational surface as shown by the dashed line in Fig. 4 (e). $\Delta \widetilde{\mathrm{T}}_{\mathrm{e}}(R)$ is calculated as:

$$
\Delta \widetilde{\mathrm{T}}_{\mathrm{e}}(R)=\frac{\left\langle\widetilde{\mathrm{T}}_{\mathrm{e}}(R, \xi)\right\rangle_{\xi}-\widetilde{\mathrm{T}}_{\mathrm{e}}^{\mathrm{ref}}(R)}{\widetilde{\mathrm{T}}_{\mathrm{e}}^{\mathrm{ref}}(R)}
$$

For clarity, we point out the difference between expressions (8) and (9). In contrast to expression (8), where the reference is a constant $\left(\widetilde{\mathrm{T}}_{\mathrm{e}}^{\mathrm{ref}}\right)$, in expression $(9)$ the reference is the helically averaged radial profile of $\widetilde{T}_{e}$ without island. This latter normalization accounts for local variations of $\widetilde{T}_{e}$ caused by ZFs at the rational surface in the simulation without island.

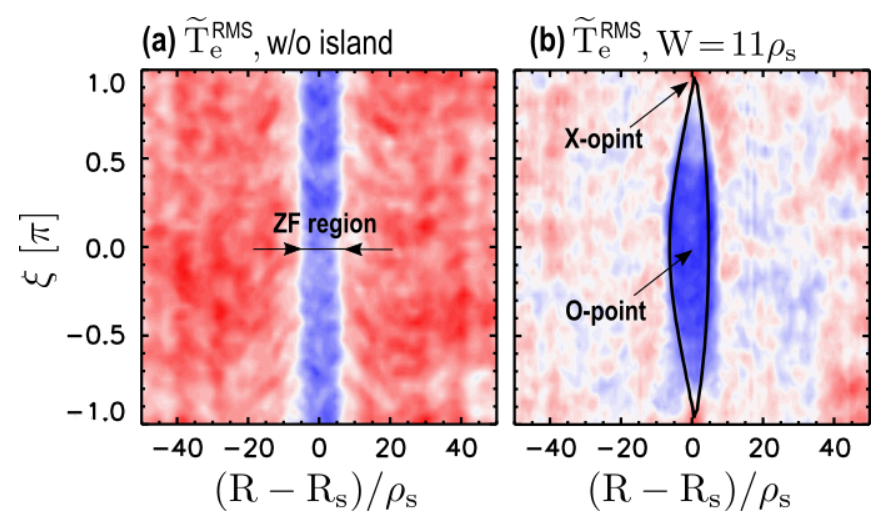

FIG. 5: Contour of the $\widetilde{\mathrm{T}}_{\mathrm{e}}$ RMS (a) w/o island and (b) with an island whose width is comparable to the radial correlation length of ZFs at $q=m / n$.

The overall picture emerging from Fig. 5 is that the 
destruction of rational flux surface topology at $q=m / n$ can significantly damp ZFs thereby allowing $\widetilde{\mathrm{T}}_{\mathrm{e}}$ increase in the $\mathrm{X}$-point region of a narrow island.

\section{DISCUSSION}

This paper reported on (i) a series of controlled $\mathrm{H}$ mode experiments in DIII-D to measure the effect of naturally occurring, freely rotating $2 / 1$ NTM islands on turbulent electron temperature fluctuations and on (ii) non-linear gyrokinetic simulations of this effect via the GENE code.

Localized measurements of low- $k \widetilde{\mathrm{T}}_{\mathrm{e}}$ via CECE, corresponding to the expected range of the Ion Temperature Gradient instability, show the following key features:

1. Reduction (increase) of $\widetilde{T}_{e}$ levels inside (outside) the region of rotating, growing and stationary $m / n=2 / 1$ magnetic islands when the island width is larger than a few times the ion gyroradius. These changes are in qualitative agreement with previously reported turbulent density fluctuation measurements ${ }^{27}$.

2. Contrary to expectation based on local gradients, $\widetilde{\mathrm{T}}_{\mathrm{e}}$ increases at $q=m / n$ when the island width is a few times the ion gyroradius. This result has been rigorously inspected by a series of analyses: (i) we have verified that this increase is present in multiple discharges, (ii) the ELM filtering and frequency filtering have no qualitative impact on this result.

3. $\widetilde{T}_{e}$ modification is radially asymmetric with weaker turbulence on the core side of the island where higher flow shear is expected.

In comparison to GENE non-linear gyrokinetic simulations with magnetic islands, qualitative agreement with the DIII-D CECE data is reported in the following characteristics:

1. $\widetilde{\mathrm{T}}_{\mathrm{e}}$ is reduced (increased) primarily at the O-point (outside) of the islands, caused by reduced (increased) local turbulence drives.

2. $\widetilde{\mathrm{T}}_{\mathrm{e}}$ increases in the island region (compared to no island) when $\mathrm{W}$ is comparable or larger to the $\mathrm{ZF}$ radial correlation length. This is caused by destroyed flux surface topology by the island which reduces ZF growth rates, reducing $\widetilde{\mathrm{T}}_{\mathrm{e}}$ regulation in the Xpoint region. Note that this effect is seen in the experiment only when $\mathrm{W}=2.8 \mathrm{~cm}$.

3. $\widetilde{\mathrm{T}}_{\mathrm{e}}$ is radially asymmetric. With $\mathrm{A}=-0.6, \widetilde{\mathrm{T}}_{\mathrm{e}}$ is smaller at $R<\mathrm{R}_{-}$compared to $\Delta \widetilde{\mathrm{T}}_{\mathrm{e}}^{\circ}$ at $\mathrm{R}_{+}<R$. This is caused by increased (reduced) flow shear at $R<\mathrm{R}_{-}\left(R<\mathrm{R}_{+}\right)$due to the radially asymmetric island flux surfaces.
These results are important as increased $\widetilde{T}_{e}$ outside of the island may explain increased thermal transport in the transient phase of NTMs ${ }^{1,56}$ while decreased $\widetilde{\mathrm{T}}_{\mathrm{e}}$ inside the island region offers explanation for measured crossfield electron thermal diffusivity $\left(\chi_{\perp}\right)$ reduction at the O-point of magnetic islands ${ }^{42,52,53}$. Observed increase of $\widetilde{\mathrm{T}}_{\mathrm{e}}$ at $q=m / n$ in the narrow island limit, potentially caused by ZF damping, may have an effect on the threshold island width for NTM growth, which should be included in NTM dynamical models, e.g. the modified Rutherford Equation. This interaction may also be related to observed increase of turbulence and transport due to resonant magnetic perturbations in ELMsuppressed plasmas ${ }^{36}$ as suggested theoretically ${ }^{34,35}$.

\section{ACKNOWLEDGEMENTS}

This material is based upon work supported by the U.S. Department of Energy, Office of Science, Office of Fusion Energy Sciences, using the DIII-D National Fusion Facility, a DOE Office of Science user facility, under Award ${ }^{1}$ DE-FC02-04ER54698. This work was supported in part by the U.S. Department of Energy under ${ }^{2}$ DE-FG02-08ER54984 and ${ }^{2}$ DE-SC0019352. The GENE gyrokinetic computations were carried out at the MARCONI-Fusion supercomputer at CINECA, Italy.

\section{DISCLAIMER}

This report was prepared as an account of work sponsored by an agency of the United States Government. Neither the United States Government nor any agency thereof, nor any of their employees, makes any warranty, express or implied, or assumes any legal liability or responsibility for the accuracy, completeness, or usefulness of any information, apparatus, product, or process disclosed, or represents that its use would not infringe privately owned rights. Reference herein to any specific commercial product, process, or service by trade name, trademark, manufacturer, or otherwise does not necessarily constitute or imply its endorsement, recommendation, or favoring by the United States Government or any agency thereof. The views and opinions of authors expressed herein do not necessarily state or reflect those of the United States Government or any agency thereof.

\section{REFERENCES}

${ }^{1}$ O. Sauter, R. J. La Haye, Z. Chang, D. A. Gates, Y. Kamada, H. Zohm, A. Bondeson, D. Boucher, J. D. Callen, M. S. Chu, T. A. Gianakon, O. Gruber, R. W. Harvey, C. C. Hegna, L. L. Lao, D. A. Monticello, F. Perkins, A. Pletzer, A. H. Reiman, M. Rosenbluth, E. J. Strait, T. S. Taylor, A. D. Turnbull, F. Waelbroeck, J. C. Wesley, H. R. Wilson, and R. Yoshinof. Beta limits in long-pulse tokamak discharges. Phys. Plasmas, 4:1654, 1997. 
${ }^{2}$ C. J. McDevitt and P. H. Diamond. Multiscale interaction of a tearing mode with drift wave turbulence: A minimal selfconsistent model. Phys. Plasmas, 13(032302), 2006.

${ }^{3}$ M. Yagi, S.-I. Itoh, K. Itoh, M. Azumi, P. H. Diamond, A. Fukuyama, and T. Hayashi. Nonlinear drive of tearing mode by microscopic plasma turbulence. Plasma and Fusion Research, 2(025), 2007.

${ }^{4}$ F. Militello, F. L. Waelbroeck, R. Fitzpatrick, and W. Horton. Interaction between turbulence and a nonlinear tearing mode in the low beta regime. Phys. Plasmas, 15(050701), 2008.

${ }^{5}$ E. Poli, A. Bottino, and A.G. Peeters. Behaviour of turbulent transport in the vicinity of a magnetic island. Nucl. Fusion, 49(075010), 2009

${ }^{6} \mathrm{H}$. R. Wilson and J W Connor. The influence of magnetic islands on drift mode stability in magnetized plasma. Plasma Phys. Control. Fusion, 51(115007), 2009.

${ }^{7}$ F L Waelbroeck, F Militello, R Fitzpatrick, and W Horton. Effect of electrostatic turbulence on magnetic islands. Plasma Phys. Control. Fusion, 51(015015), 2009.

${ }^{8}$ W A Hornsby, M Siccinio, A G Peeters, E Poli, A P Snodin, F J Casson, Y Camenen, and G Szepesi. Interaction of turbulence with magnetic islands: effect on bootstrap current. Plasma Phys. Control. Fusion, 53(054008), 2011.

${ }^{9}$ W. A. Hornsby, A. G. Peeters, M. Siccinio, and E. Poli. On the dynamics of vortex modes within magnetic islands. Phys. Plasmas, 19(032308), 2012.

${ }^{10} \mathrm{~A}$. Ishizawa and F. L. Waelbroeck. Magnetic island evolution in the presence of ion-temperature gradient-driven turbulence. Phys. Plasmas, 20(122301), 2013.

${ }^{11}$ O. Agullo, M. Muraglia, A. Poye, S. Benkadda, M. Yagi, X. Garbet, and A. Sen. A signature of turbulence driven magnetic islands. Phys. Plasmas, 21(092303), 2014.

${ }^{12}$ Z. Q. Hu, Z. X. Wang, L. Wei, J. Q. Li, and Y. Kishimoto. Nonlinear mutual destabilization of the tearing mode and ion temperature gradient mode. Nucl. Fusion, 54(123018), 2014.

${ }^{13}$ D. Zarzoso, W.A. Hornsby, E. Poli, F.J. Casson, and A.G. Peetersand S. Nasr. Impact of rotating magnetic islands on density profile flattening and turbulent transport. Nucl. Fusion, 55(113018), 2015.

${ }^{14} \mathrm{P}$. Hill, F. Hariri, and M. Ottaviani. The effect of magnetic islands on ion temperature gradient turbulence driven transport. Phys. Plasmas, 22(042308), 2015.

${ }^{15}$ W. A. Hornsby, P. Migliano, R. Buchholz, D. Zarzoso, F. J. Casson, E. Poli, and A. G. Peeters. On seed island generation and the non-linear self-consistent interaction of the tearing mode with electromagnetic gyro-kinetic turbulence. Plasma Phys. Control. Fusion, 57(054018), 2015.

${ }^{16}$ W. A. Hornsby, P. Migliano, R. Buchholz, L. Kroenert, A. Weikl, A. G. Peeters, D. Zarzoso, E. Poli, and F. J. Casson. The linear tearing instability in three dimensional, toroidal gyro-kinetic simulations. Phys. Plasmas, 22(022118), 2015.

${ }^{17}$ Olivier Izacard, Christopher Holland, Spencer D. James, and Dylan P. Brennan. Dynamics of ion temperature gradient turbulence and transport with a static magnetic island. Phys. Plasmas, 23(022304), 2016

${ }^{18}$ A. Bañón Navarro, L. Bardóczi, T. A. Carter, F. Jenko, and T. L. Rhodes. Effect of magnetic islands on profiles, flows, turbulence and transport in nonlinear gyrokinetic simulations. Plasma Physics and Controlled Fusion, 59(3):034004, 2017.

${ }^{19}$ R. E. Waltz and F. L. Waelbroeck. Gyrokinetic simulations with external resonant magnetic perturbations: Island torque and nonambipolar transport with plasma rotation. Phys. Plasmas, 19(3), 2012

${ }^{20}$ C. $\mathrm{Yu}$, D. L. Brower, S.Zhao, R.V. Bravenec, J. Chen, H. Lin, N.C. Luhamann Jr., W.A. Peebles, C.P. Ritz, P.M. Schoch, and $\mathrm{X}$. Yang. Tearing instabilities and microturbulence in TEXT. Nucl. Fusion, 32(9):1545, 1992

${ }^{21}$ K.J. Zhao, Y.J. Shi, S.H. Hahnand P.H. Diamond, Y. Sun 5, J. Cheng, H. Liu, N. Lie, Z.P. Chen, Y.H. Ding, Z.Y. Chen, B. Rao, M. Leconte, J.G. Bak, Z.F. Cheng, L. Gao, X.Q. Zhang,
Z.J. Yang, N.C. Wang, L. Wang, W. Jin, L.W. Yan, J.Q. Dong, G. Zhuang, and J-TEXT team. Plasma flows and fluctuations with magnetic islands in the edge plasmas of J-TEXT tokamak. Nucl. Fusion, 55(073022), 2015.

${ }^{22}$ T. Estrada, E. Ascasibar, E. Blanco, A. Cappa, C. Hidalgo, K. Ida, A. Lopez-Fraguas, and B. Ph van Milligen. Plasma flow, turbulence and magnetic islands in TJ-II. Nucl. Fusion, 56(026011), 2016

${ }^{23}$ K. Ida, T. Kobayashi, M. Ono, T. E. Evans, G. R. McKee, and M. E. Austin. Hysteresis Relation between Turbulence and Temperature Modulation during the Heat Pulse Propagation into a Magnetic Island in DIII-D. Phys. Rev. Lett., 120(245001), 2018.

${ }^{24}$ K.J. Zhao, Y. Nagashima, F.M. Li, Yuejiang Shi, P.H. Diamond, J.Q. Dong, K. Itoh, S.-I. Itoh, G. Zhuang, H. Liu, Z.P. Chen, J. Cheng, L. Nie, Y.H. Ding, Q.M. Hu, Z.Y. Chen, B. Rao, Z.F. Cheng, L. Gao, X.Q. Zhang, Z.J. Yang, N.C. Wang, L. Wang, W. Jin, W. Yan, J.Q. Xu, Y.F. Wu, L.W. Yan, A. Fujisawa, S. Inagaki, Y. Kosuga, M. Sasaki, and J-TEXT Team. Temporalspatial structures of plasmas flows and turbulence around tearing mode islands in the edge tokamak plasmas. Nuclear Fusion, 57(12):126006, 2017

${ }^{25}$ L. Bardóczi, T. L. Rhodes, A. Bañón Navarro, C. Sung, T. A. Carter, R. J. La Haye, C. Petty, C. Crystal, and F. Jenko. Impact of Neoclassical Tearing Mode - turbulence multi-scale interaction in confinement degradation and magnetic island stability. Phys. Plasmas, 24(122503), 2017.

${ }^{26}$ L. Bardóczi, T. L. Rhodes, T. A. Carter, A. Bañón Navarro R. J. La Haye, and G. R. McKee. Shrinking of core Neoclassical Tearing Mode magnetic islands due to Edge Localized Modes and the role of ion-scale turbulence in island recovery in DIII-D. Phys. Plasmas, 24(062503), 2017.

${ }^{27}$ L. Bardóczi, T. L. Rhodes, A. Bañón Navarro, C. Sung, T. A. Carter, R. J. La Haye, C. Petty, C. Crystal, and F. Jenko. Multifield/-scale Interactions of Turbulence with Neoclassical Tearing Mode magnetic islands in the DIII-D Tokamak. Phys. Plasmas, 24(056106), 2017.

${ }^{28}$ L. Bardóczi, T. L. Rhodes, T. A. Carter, A. Bañón Navarro, W. A. Peebles, F. Jenko, and G. McKee. Modulation of Core Turbulent Density Fluctuations by Large-Scale Neoclassical Tearing Mode Islands in the DIII-D Tokamak. Phys. Rev. Lett., 116:215001, May 2016

${ }^{29}$ M.J. Choi, J. Kim, J.-M. Kwon, H.K. Park, Y. In, W. Lee, K.D. Lee, G.S. Yun, J. Lee, M. Kim, W.-H. Ko, J.H. Lee, Y.S. Park, Y.-S. Na, N.C. Luhmann Jr, and B.H. Park. Multiscale interaction between a large scale magnetic island and small scale turbulence. Nuclear Fusion, 57(12):126058, 2017.

${ }^{30}$ M. Jiang, Y. Xu2, W. Chen, Z. B. Shi, J. Q. Li, W. X. Wang, Z. H. Qin, X. T. Ding, W. L. Zhong, X. Q. Ji, P. W. Shi, Z. C. Yang, B. S. Yuan, Y. Liu, Q. W. Yang, M. Xu, and the HL2A Team. Localized modulation of turbulence by $\mathrm{m} / \mathrm{n}=1 / 1$ magnetic islans in the HL-2A tokamak. Nucl. Fusion, (accepted)(102662), 2019.

${ }^{31}$ J. L. Luxon. A design retrospective of the DIII-D tokamak. Nucl. Fusion, 42(614), 2002.

${ }^{32}$ C. Sung, W. A. Peebles, C. Wannberg, T. L. Rhodes, X. Nguyen, R. Lantsov, and L. Bardóczi. A frequency tunable, eightchannel correlation ECE system for electron temperature turbulence measurements on the DIII-D tokamak. Rev. Sci. Instrum., 87(11E123), 2016.

${ }^{33}$ F. Jenko, W. Dorland, M. Kotschenreuther, and B. N. Rogers. Electron temperature gradient driven turbulence. Phys. Plasmas, 7(1904), 2000

${ }^{34} \mathrm{M}$. Leconte and P. H. Diamond. Effect of resonant magnetic perturbations on secondary structures in drift-wave turbulence. Phys. Plasmas, 18(082309), 2011.

${ }^{35}$ T. M. Bird and C. C. Hegna. A model for microinstability destabilization and enhanced transport in the presence of shielded 3D magnetic perturbations. Nucl. Fusion, 53(013004), 2013.

${ }^{36}$ G.R. McKee, Z. Yan, C. Holland, R.J. Buttery, T.E. Evans, R.A. Moyer, S. Mordijck, R. Nazikian, T.L. Rhodes, O. Schmitz, and 
M.R. Wade. Increase of turbulence and transport with resonant magnetic perturbations in ELM-suppressed plasmas on DIII-D. Nucl. Fusion, 53(113011), 2013.

${ }^{37}$ L. Bardóczi. Multi-Field/-Scale Interaction of Neoclassical Tearing Modes with Turbulence and Impact on Plasma Confinement. PhD thesis, University of California, Los Angeles, 2017.

${ }^{38}$ M. E. Austin and J. Lohr. Electron cyclotron emission radiometer upgrade on the DIII-D tokamak. Rev. Sci. Instrum., 74:1457, 2003.

${ }^{39}$ K. H. Burrell, P. Gohil, R. J. Groebner, D. H. Kaplan, J. I. Robinson, and W. M. Solomon. Improved charge-coupled device detectors for high-speed, charge exchange spectroscopy studies on the DIII-D tokamak. Rev. Sci. Instrum, 75(10):3455, 2004.

${ }^{40}$ M. A. Van Zeeland, R. L. Boivin, T. N. Carlstrom, T. Deterly, and D. K. Finkenthal. Fiber optic two-color vibration compensated interferometer for plasma density measurements. Rev. Sci. Instrum, 77(10F325), 2006.

${ }^{41} \mathrm{D}$ De Lazarri and $\mathrm{E}$ Westerhof. The role of asymmetries in the growth and suppression of neoclassical tearing modes. Plasma Phys. Control. Fusion, 53(035020), 2011.

${ }^{42}$ L. Bardóczi, T. L. Rhodes, T. A. Carter, N. A. Crocker, W. A. Peebles, and B. A. Grierson. Non-perturbative measurement of cross-field thermal diffusivity reduction at the O-point of $2 / 1$ neoclassical tearing mode islands in the DIII-D tokamak. Phys. Plasmas, 23(052507), 2016.

${ }^{43}$ L. Bardóczi, M. Podestà, W. W. Heidbrink, and M. A. Van Zeeland. Quantitative modeling of neoclassical tearing mode driven fast ion transport in integrated transp simulations. Plasma Phys. Control. Fusion, (submitted), 2009.

${ }^{44}$ G. G. Plunk, P. Helander, P. Xanthopoulos, and J. W. Connor. Collisionless microinstabilities in stellarators. III. The iontemperature-gradient mode. Phys. Plasmas, 21(032112), 2014.

${ }^{45}$ F. Jenko, W. Dorland, and G. W. Hammett. Critical gradient formula for toroidal electron temperature gradient modes. Phys. Plasmas, 8(4096), 2001.

${ }^{46}$ P. Migliano, P. Mantica, B. Baiocchi, A. Casati, J. Citrin, C. Giround, N. Hawkes, E. Lerche, M. Tsalas, D. Van Eester, and JET EFDA Contributors. The dependence of ion heat transport on the ion to electron temperature ratio in jet non-rotating plasmas. Plasma Phys. Control. Fusion, 55(055003), 2013.

${ }^{47}$ T. N. Carlstrom, G. L. Campbell, J. C. DeBoo, R. Evanko, J. Evans, C. M. Greenfield, J. Haskovec, C. L. Hsieh, E. Mc-
Kee, R. T. Snider, R. Stockdale, P. K. Trost, and M. P. Thomas. Design and operation of the multipulse Thomson scattering diagnostic on DIII-D. Rev. Sci. Instrum, 63(10):4901-4906, 1992.

${ }^{48} \mathrm{C}$. Watts. A review of ece correlation radiometry techniques for detection of core electron temperature fluctuations. Fusion Science and Technology, 52(176), 2007.

${ }^{49}$ G. Cima, R. V. Bravenec, A. J. Wootton, T. D. Rempel, R. F. Gandy, C. Watts, and M. Kwon. Core temperature fluctuations and related heat transport in the texas experimental tokamak upgrade. Phys. Plasmas, 2(720), 1995.

${ }^{50}$ T. D. Rempel, R. F. Gandy, and A. J. Wootton. Density fluctuation effects on electron cyclotron emission correlation measurements in optically gray plasmas. Rev. Sci. Instrum., 2044(65), 1994.

${ }^{51}$ Ch. Fuchs and M. E. Austin. Measurements of edge-localizedmode induced electron cyclotron emission bursts in DIII-D. Phys. Plasmas, 8(1594), 2001.

${ }^{52}$ I. G. J. Classen, E. Westerhof, C. W. Domier, A. J. H. Donne, R. J. E. Jaspers, N. C. Luhmann, Jr., H. K. Park, M. J. van de Pol, G. W. Spakman, M. W. Jakubowski, and the TEXTOR team. Effect of Heating on the Suppression of Tearing Modes in Tokamaks. Phys. Rev. Lett., 98:035001, 2007.

${ }^{53}$ K. Ida, K. Kamiya, A. Isayama, Y. Sakamoto, and JT-60 Team. Reduction of ion thermal diffusivity inside a magnetic island in jt-60u tokamak plasma. Phys. Rev. Lett., 109(065001), 2012.

${ }^{54}$ A. M. Dimits, G. Bateman, M. A. Beer, B. I. Cohen, W. Dorland, G. W. Hammett, C. Kim, J. E. Kinsey, M. Kotschenreuther, A. H. Kritz, L. L. Lao, J. Mandrekas, W. M. Nevins, S. E. Parker, A. J. Redd, D. E. Shumaker, R. Sydora, and J. Weiland. Comparisons and physics basis of tokamak transport models and turbulence simulations. Phys. Plasmas, 7(969), 2000.

${ }^{55}$ W. A. Hornsby, A. G. Peeters, A. P. Snodin, F. J. Casson, Y. Camenen, G. Szepesi, M. Siccinio, and E. Poli. The nonlinear coupling between gyroradius scale turbulence and mesoscale magnetic islands in fusion plasmas. Phys. Plasmas, 17(092301), 2010.

${ }^{56}$ Z. Chang and J. D. Callen. Global energy confinement degradation due to macroscopic phenomena in tokamaks. Nucl. Fusion, 30(2):219, 1990. 